\title{
Development and Psychometric Properties of the Japanese Heart Failure Knowledge Scale Japanese Heart Failure Knowledge Scale
}

\author{
Naoko Kato, ${ }^{1} \mathrm{PhD}$, Koichiro Kinugawa, ${ }^{2} \mathrm{MD}$, Etsuko NaKayama, ${ }^{3}$ Akiko Hatakeyama, ${ }^{3}$ \\ Takako Tsuji, ${ }^{3}$ Yumiko Kumagai, ${ }^{3}$ Issei Komuro, ${ }^{1} \mathrm{MD}$, and Ryozo NaGAI, ${ }^{4} \mathrm{MD}$
}

\begin{abstract}
SUMMARY
Knowledge about their own condition is important for patients with heart failure (HF). No valid, reliable, and easily administered instrument is available to measure this knowledge in clinical practice. In this study, a HF knowledge scale was developed, and its psychometric properties were tested. Items related to knowledge about HF were extracted from relevant guidelines. Content validity of the items was confirmed by an expert panel including a cardiologist and nurses specialized in treatment and care of patients with HF. A self-administered questionnaire was then distributed to $187 \mathrm{pa}-$ tients with HF $(64.0 \pm 12.1$ years, males $69 \%)$. In $62 \%$ patients, a left ventricular ejection fraction of $<50 \%$ was identified. Exploratory factor analysis demonstrated the one-dimensionality of the 15-item HF knowledge scale. Mean score was $10.7 \pm 3.0$ (range, $0-15$ ). Known-group validity testing revealed a significant difference in HF knowledge score between patients newly diagnosed with $\mathrm{HF}$ and patients experienced with $\mathrm{HF}(9.4 \pm 3.2$ versus $10.8 \pm 2.9, P=0.043)$. In addition, HF knowledge scale scores were correlated with HF self-care scores assessed by the European Heart Failure Self-Care Behavior Scale for evaluation of criterion validity $(\rho=-0.304, P<0.001)$. Cronbach's alpha was 0.79 , and item-total correlation was $0.22-0.51$, thereby suggesting that the reliability of the scale was acceptable. Acceptable validity and reliability were demonstrated for the HF knowledge scale developed in this study. This instrument could be useful in evaluation of patient knowledge about HF. (Int Heart J 2013; 54: 228-233)
\end{abstract}

Key words: Patient compliance, Patient education, Questionnaire, Reliability, Self-care, Validity

$\mathrm{W}$ ith the rapid aging of society in both Japan and Western countries, the number of patients with HF has also increased. ${ }^{1)}$ Despite improvements in HF therapy, high rates of rehospitalization persist. In Japan, approximately $20 \%$ patients with $\mathrm{HF}$ were rehospitalized within 1 year of discharge. ${ }^{2,3)} \mathrm{HF}$ creates physiological and psychological burdens for patients and their families, and also places an enormous economic burden on the health insurance system. Therefore, preventing deterioration of this disease in patients with chronic HF is extremely important.

In some cases, rehospitalization in patients with HF has been caused by their lack of self-care or inadequate knowledge about diet, medication, and HF symptoms. ${ }^{477}$ A meta-analysis revealed a significant reduction in the rate of hospitalization due to worsening HF because of disease management programs aimed at enhancing self-care. ${ }^{8)}$ Although the underlying mechanism of this improved rate of hospitalization was not specified, the increase in knowledge about their own condition in patients with HF may have contributed to these results, thereby enhancing self-care practice and improving clinical outcome. HF knowledge does not always guarantee good adherence to a therapeutic regimen, but previous studies have indicated that better adherence is often associated with greater knowledge. ${ }^{9-12)}$ Considering the fact that knowledge about HF may have a positive influence on patient prognosis, appropriate evaluation of the extent to which patients understand their condition may aid health care professionals in improving treatment adherence as well as outcome in patients with HF.

A review of the literature revealed that 4 instruments are currently available for measurement of the levels of HF knowledge. Two of these are questionnaires with comprehensive coverage of HF topics such as symptoms and treatment of $\mathrm{HF}^{9,13)}$ However, the validity and reliability of these scales has not been fully demonstrated. Reilly, et al developed the Atlanta Heart Failure Knowledge test. ${ }^{14)}$ Although the content and construct validities and internal consistency of this test have been confirmed, the assessment length constitutes a barrier to its easier application in clinical settings. It contains 30 multiple-

From the Departments of ${ }^{1}$ Cardiovascular Medicine and ${ }^{2}$ Therapeutic Strategy for Heart Failure, The University of Tokyo Graduate School of Medicine, ${ }^{3}$ Department of Nursing, Sakakibara Heart Institute, Tokyo, and ${ }^{4}$ Jichii Medical University, Tochigi, Japan

This work was supported in part by a Grant-in-Aid for the JSPS Postdoctoral Research Fellow from the Japan Society for the Promotion of Science (No. 224943) to N.K., Japanese Heart Foundation, Japanese Association for Cerebro-cardiovascular Disease Control, and AstraZeneca for clinical epidemiology research to N.K and K.K., and a domestic collaborative research grant from the Pfizer Health Research Foundation to N.K. and K.K.

Address for correspondence: Naoko Kato, PhD, Department of Cardiovascular Medicine, The University of Tokyo Graduate School of Medicine, 7-3-1 Hongo, Bunkyo-ku, Tokyo 113-8655, Japan.

Received for publication February 12, 2013.

Revised and accepted March 14, 2013. 
choice items and requires considerable time to complete. Finally, the psychometric properties of the 15 -item Dutch Heart Failure Knowledge Scale have been confirmed. ${ }^{15)}$ The scale has been developed for research purposes, not for use in clinical practice. As a result, the questions on this instrument may require clinical knowledge that is too advanced for most $\mathrm{HF}$ patients.

A valid, reliable, and easily administered scale must be available for the measurement of knowledge in patients with HF; subsequently, test results of such scales may be used to enhance self-care practice and improve clinical outcomes. In this study, a HF knowledge scale was developed, and its validity and reliability were tested.

\section{Methods}

Study procedure: A new instrument was developed in the form of a self-administered questionnaire for testing knowledge about HF in patients with HF. The content validity of the instrument was assessed before conducting the main study using focus group analysis. Moreover, a pilot study was conducted to determine face validity of the instrument. To test the psychometric properties of the HF knowledge scale, the questionnaire was distributed and scored against a master copy containing correct answers to all the items. Each item of the new scale was assessed for skewed distributions. Thereafter, construct and criterion validity analyses were performed. In addition, the sensitivity and reliability of the new scale were evaluated.

Instrument development: To develop an instrument for measuring knowledge about HF, HF guidelines ${ }^{16-18)}$ and prior studies ${ }^{9,10,12,19-23)}$ were consulted. The guidelines stated that HF patients should have at least some general knowledge of HF, its signs and symptoms, related treatment, and self-care. Based on these guidelines and the results of earlier studies regarding knowledge about HF and its effects on patient outcome, a new HF knowledge scale was developed initially containing 17 items concerning HF in general (3 items; eg, "HF is a condition in which the heart is not able to pump blood through the body in sufficient amounts"), HF symptoms and signs (5 items; eg, "difficulty in breathing and shortness of breath are symptoms of HF"), and HF-related treatment and self-care (9 items; eg, "diuretics remove fluids from the body" and "HF patients had better take a high-salt diet"). Several self-care-related items such as medication, fluid intake, and exercise were selected based on common misconceptions or concerns identified in HF patients in other studies. ${ }^{10,12,19-23)}$

The items on the scale were represented by questions to which patients could respond with "yes," "no," or "I do not know." One point was given for each correct answer; an incorrect answer or an answer of "I do not know" received no points. The total knowledge score was determined by the sum of these points, and a higher score indicated greater knowledge about HF.

Pilot study: To ensure that the instrument was comprehensible to patients with HF, several patients were asked to complete the questionnaire and provide feedback about difficulty interpreting the questions. In addition, the time required to answer the questions was recorded.

Study patients: All consecutive patients with HF hospitalized between March 2009 and March 2010 and consecutive outpatients with HF who had a history of hospitalization due to worsening HF between October 2010 and October 2011 were enrolled from two independent hospitals. The exclusion criteria were: age $>20$ years, physical inability to complete the questionnaire, serious psychological distress (as determined by the primary physician), or unwillingness to provide informed consent. Three research nurses selected the patients who met these criteria, and asked them to complete the questionnaire.

All patients provided informed consent after the purpose of the study was thoroughly explained. The institutional review boards of the University of Tokyo (No. 2145, July, 2008) and Sakakibara Heart Institute (September, 2010) approved this study.

Content validity: The content validity of the new HF knowledge scale was discussed in a focus group including a cardiologist, 3 expert nurses, and a nursing researcher; all focus group participants were specialists in the area of HF. To assess its content validity, the following questions were asked: (1) was the new HF knowledge scale easy to understand and complete? (2) was the new HF knowledge scale applicable to patients with HF?

Construct validity: One approach to construct validation is the known-group technique, which tests the ability of an instrument to distinguish between two groups of patients, who are assumed to have different scores on an instrument. We hypothesized that patients newly diagnosed with HF would have lower knowledge scores than those experienced with HF on the basis of results of prior studies. ${ }^{20,23)}$ In the participating institutions, when patients are hospitalized and diagnosed with $\mathrm{HF}$, they receive an educational intervention about HF during hospitalization. In this study, if patients were newly diagnosed with HF, they were asked to complete the HF knowledge scale before the intervention. Thereafter, the known-group technique was utilized to compare the scores of newly diagnosed patients with those of more experienced HF patients to determine the construct validity of the scale.

Criterion validity: Because no scale has been available to measure patient knowledge about HF in Japan for comparison with the scale developed here, the relationship between HF knowledge and HF-related self-care, a concept theoretically linked with HF knowledge, was examined in this study. ${ }^{9-12,24)}$ We assumed an association between greater HF knowledge and better self-care. Self-care behavior was assessed using the Japanese version of the European Heart Failure Self-Care Behavior Scale (EHFScBS). ${ }^{25,26)}$ This is a 12-item self-administered questionnaire that obtains information on the self-care behavior of patients with $\mathrm{HF}$, including daily weighing, fluid restriction, and medication. For each item, patients rate their self-care behavior on a 5-point scale from 1 ("I completely agree") to 5 ("I do not agree at all"). The total score ranges from 12 to 60, and a higher score indicates poorer self-care behavior. The validity and reliability of the EHFScBS have been confirmed in a previous study. ${ }^{25}$ )

Sensitivity: The sensitivity of an instrument reflects its ability to detect small variations in knowledge. In this study, differences in knowledge score before and after the HF educational intervention were investigated. Ten patients were included in a subgroup analysis of sensitivity. Subjects were patients hospitalized due to worsening HF, who received comprehensive education about HF, including published material about the con- 
dition, ${ }^{22)}$ from nurses and pharmacists during hospitalization. One month after discharge, the questionnaire was administered to these patients in order to evaluate the levels of HF knowledge.

Demographic and clinical parameters: The following characteristics of the subjects were investigated: gender, age, marital status, employment, etiology of HF, prior hospitalization with HF, B-type natriuretic peptide (BNP) levels, left ventricular ejection fraction, New York Heart Association functional class, systolic blood pressure, heart rate, comorbidity such as diabetes mellitus and chronic kidney disease (defined as estimated glomerular filtration rate $<60 \mathrm{~mL} /$ minute $/ 1.73 \mathrm{~m}^{2}$ ) calculated using the Japanese formula, ${ }^{27)}$ and medication. These data were collected from patient medical records.

Statistical analysis: Categorical data are presented as frequencies and percentages. For continuous variables with a normal distribution, the mean and standard deviation are reported. For BNP, logarithmic transformation was performed and data were analyzed using parametric methods. Values of BNP levels are presented as antilogarithms. To assess factor validity, exploratory factor analysis was performed using the principal component analysis extraction method. The number of factors was determined using a scree plot based on an eigenvalue threshold of 1.0 or higher, and total variance explained was determined. For identifying variables associated with a given factor, 0.35 was used as a cutoff point for defining variables associated with a given factor. Known-group validity was examined with Student's $t$-test to compare the scores of two groups. To examine criterion validity, Spearman's correlation coefficient was calculated to determine correlations between HF knowledge scale scores and EHFScBS scores. Internal consistency and item-total correlation were assessed with Cronbach's alpha coefficient and Pearson product-moment correlation, respectively.

All statistical tests were two-tailed, and $P$-values $<0.05$ were considered statistically significant. Statistical analysis was performed using SAS version 9.3 for Windows (SAS Institute, Cary, NC, USA).

\section{RESULTS}

Face validity: A pilot test was performed involving 6 patients (3 males and 3 females) with HF. Their mean age was $61.5 \pm$ 20.0 years (range, 35-82 years). None of the patients reported any difficulty in answering or interpreting the questions on the HF knowledge questionnaire. None of the items were missing in the responses to the scale. The time taken to answer all questions on the HF knowledge questionnaire was about 5 minutes. On the basis of these results, the HF knowledge scale was used for the subsequent validation study.

Characteristics of study patients: Of 190 patients who had agreed to participate in the survey, 187 were included in the study. Three patients were excluded due to missing items on their questionnaires ( $>40 \%$ of all questions). Patient characteristics are presented in Table I. The mean age of the participants was $64.0 \pm 12.1$ years, with $69 \%$ male respondents. In $26 \%$ of the patients, the disease was of ischemic etiology, whereas in $62 \%$ of the patients, a left ventricular ejection fraction of < $50 \%$ was identified. The mean value of BNP levels was 194.4 $\mathrm{pg} / \mathrm{mL}$. With regard to medication, beta-blockers and angiotensin-converting enzyme inhibitors and/or angiotensin re-
Table I. Characteristics of Study Patients $(n=187)$

\begin{tabular}{|c|c|}
\hline Age, years & $64 \pm 12.1$ \\
\hline Gender, male & $129(69 \%)$ \\
\hline \multicolumn{2}{|l|}{ Married status } \\
\hline Unmarried & $22(12 \%)$ \\
\hline Married & $136(74 \%)$ \\
\hline Divorced/widowed & $24(13 \%)$ \\
\hline Other & $3(1.6 \%)$ \\
\hline Employment, employed & $83(44 \%)$ \\
\hline \multicolumn{2}{|l|}{ Etiology of HF } \\
\hline Ischemic & $48(26 \%)$ \\
\hline Cardiomyopathy & $55(29 \%)$ \\
\hline Valvular heart disease & $38(20 \%)$ \\
\hline Hypertension & $9(4.8 \%)$ \\
\hline Other & $37(20 \%)$ \\
\hline Prior hospitalization for $\mathrm{HF}$ & $165(88 \%)$ \\
\hline BNP, pg/mL & $194.4 \pm 3.3$ \\
\hline LVEF, \% & $43.3 \pm 14.8$ \\
\hline $\mathrm{LVEF}<50 \%$ & $116(62 \%)$ \\
\hline NYHA class, I or II & $165(88 \%)$ \\
\hline Systolic BP, mmHg & $117.9 \pm 23.0$ \\
\hline Heart rate, beats/minute & $70.6 \pm 12.5$ \\
\hline Diabetes mellitus & $52(28 \%)$ \\
\hline Atrial fibrillation & $84(45 \%)$ \\
\hline Chronic kidney disease & $102(55 \%)$ \\
\hline \multicolumn{2}{|l|}{ Medication } \\
\hline$\beta$-Blocker & $161(86 \%)$ \\
\hline ACEI and/or ARB & $131(70 \%)$ \\
\hline Diuretics & $127(68 \%)$ \\
\hline Aldosterone blocker & $90(48 \%)$ \\
\hline HF knowledge scale score ${ }^{*}$ & $10.7 \pm 3.0$ \\
\hline $\begin{array}{l}\text { European Heart Failure Self-care Behavior } \\
\text { Scale score }\end{array}$ & $31.7 \pm 9.9$ \\
\hline
\end{tabular}

HF indicates heart failure; NYHA, New York Heart Association; BNP, Btype natriuretic peptide; $\mathrm{LVEF}$, left ventricular ejection fraction; $\mathrm{BP}$, blood pressure; ACEI, angiotensin-converting-enzyme inhibitor; and ARB, angiotensin II receptor blocker. Values are $n(\%)$ or mean \pm standard deviation. ", A higher score indicates that patients have greater level of knowledge about HF. Score ranges from 0 to $15 .{ }^{\dagger}$, A higher score indicates poorer self-care behavior. Score ranges from 12 to 60 .

ceptor blockers were prescribed in $86 \%$ and $70 \%$ of the patients, respectively.

Validity and reliability of the HF knowledge scale: Table II presents the pooled items of the first version of the HF knowledge scale. The percentages of correct answers ranged from $16 \%$ to $95 \%$. Exploratory factor analysis including 17 items demonstrated one-dimensionality of the scale in terms of the eigenvalue, the scree plot, and the total variance explained. Moderate to strong factor loading ( $>0.35)$ was evident for all items except the following 3: item 1 ("the heart has two chambers," factor loading = 0.291), item 7 ("blood-speckled sputum is characteristic of worsening HF," factor loading $=0.156$ ), and item 12 ("HF patients are discouraged from taking medications without food," factor loading $=0.272$ ). Because of their low factor loadings, items 1 and 7 were eliminated from the scale. With regard to item 12, a previous study indicated strong misconceptions about medication regimens in patients with HF. ${ }^{11,19,28)}$ Therefore, this item was included in view of its clinical importance. ${ }^{6,11,29)}$ After the two items had been eliminated, the contribution of one factor in explaining the variation in the item pool was $71 \%$. Factor loadings of each item ranged from 0.259 (item 12) to 0.594 (item 14). The mean score was $10.7 \pm$ 3.0 (range, 0-15). 
Table II. Distributions of the Item Response $(n=187)$

\begin{tabular}{|c|c|c|c|c|c|}
\hline \multirow{2}{*}{\multicolumn{2}{|c|}{ Item }} & \multirow{2}{*}{$\begin{array}{l}\% \text { of correct } \\
\text { answer }\end{array}$} & \multicolumn{3}{|c|}{ Distributions of the item response } \\
\hline & & & yes & no & I don't know \\
\hline \multicolumn{6}{|c|}{ HF in general } \\
\hline 1 & The heart has two chambers & $25 \%$ & $64 \%$ & $25 \%$ & $11 \%$ \\
\hline 2 & Exchange of oxygen and carbon dioxide occurs in heart & $33 \%$ & $38 \%$ & $33 \%$ & $29 \%$ \\
\hline 3 & $\begin{array}{l}\text { HF is a condition in which the heart is not able to pump blood through the body in suffi- } \\
\text { cient amounts }\end{array}$ & $90 \%$ & $90 \%$ & $1.6 \%$ & $8.6 \%$ \\
\hline \multicolumn{6}{|c|}{$\underline{\text { HF symptoms and signs }}$} \\
\hline 4 & Difficulty in breathing and shortness of breath are symptoms of HF & $95 \%$ & $95 \%$ & $2.1 \%$ & $2.7 \%$ \\
\hline 5 & One of the symptoms when the lungs become congested with fluid is shortness of breath & $59 \%$ & $59 \%$ & $8.0 \%$ & $33 \%$ \\
\hline 6 & $\begin{array}{l}\text { Some patients with severe HF become breathless when they lie flat and feel much better } \\
\text { when they sit up }\end{array}$ & $47 \%$ & $20 \%$ & $47 \%$ & $33 \%$ \\
\hline 7 & Blood-speckled sputum is characteristic of worsening HF & $16 \%$ & $16 \%$ & $22 \%$ & $61 \%$ \\
\hline 8 & Short-term weight gain is one of the signs of worsening HF & $56 \%$ & $56 \%$ & $8.6 \%$ & $36 \%$ \\
\hline \multicolumn{6}{|c|}{ HF related treatment and self-care } \\
\hline 9 & Overwork and stress sometimes cause HF to get worse & $79 \%$ & $79 \%$ & $1.1 \%$ & $19 \%$ \\
\hline 10 & Sodium causes water retention & $63 \%$ & $63 \%$ & $4.8 \%$ & $32 \%$ \\
\hline 11 & Diuretics remove fluids from the body & $85 \%$ & $85 \%$ & $3.7 \%$ & $11 \%$ \\
\hline & HF patients are discouraged from taking medications without food & $52 \%$ & $21 \%$ & $52 \%$ & $27 \%$ \\
\hline 13 & HF patients had better drink more water than healthy people & $67 \%$ & $11 \%$ & $67 \%$ & $22 \%$ \\
\hline 14 & HF patients had better take a high-salt diet & $92 \%$ & $0.5 \%$ & $92 \%$ & $7.5 \%$ \\
\hline 15 & Smoking is good for patients with HF because it promotes the circulation of blood & $91 \%$ & $3.7 \%$ & $91 \%$ & $4.8 \%$ \\
\hline 16 & HF patients should not perform exercise regardless of their severity of HF & $71 \%$ & $8.6 \%$ & $71 \%$ & $20 \%$ \\
\hline 17 & HF patients had better take a hot bath to promote blood circulation & $86 \%$ & $2.7 \%$ & $86 \%$ & $11 \%$ \\
\hline
\end{tabular}

HF indicates heart failure.
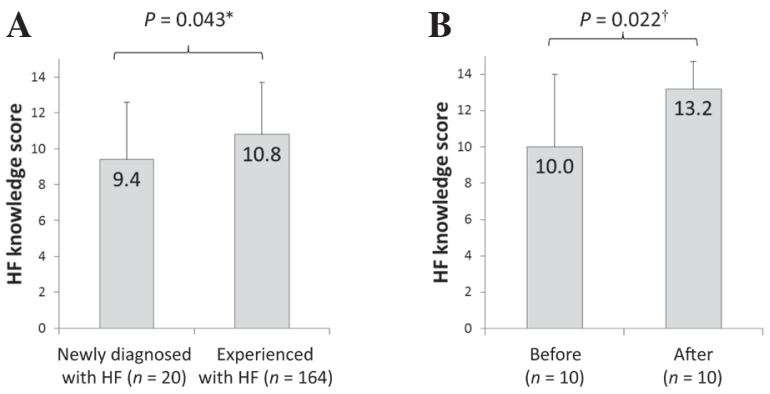

Figure 1. Known-group validity (A) and sensitivity for the Japanese heart failure knowledge scale (B). A higher HF knowledge score indicates greater level of knowledge about HF. HF indicates heart failure. " by unpaired $t$-test, ${ }^{\dagger}$ by paired $t$-test.

In the analysis of known-group validity, 20 patients had been newly diagnosed with HF. Significantly lower scores were observed for these patients than for those experienced with $\mathrm{HF}(9.4 \pm 3.2$ versus $10.8 \pm 2.9, P=0.043$; Figure $1 \mathrm{~A})$. The mean EHFScBS score for criterion validity was $31.7 \pm 9.9$. Spearman's rank correlation coefficient between HF knowledge scores and EHFScBS scores was $-0.304(P<0.001)$.

Regarding sensitivity of the knowledge scale, 10 hospitalized patients with HF received comprehensive education about HF during hospitalization. Their mean knowledge score one month after discharge was significantly higher than the baseline scores $(10.0 \pm 4.0$ versus $13.2 \pm 1.5, P=0.022$; Figure 1B).

Cronbach's alpha for internal consistency was 0.79. As displayed in Table III, the internal consistency of the instrument could not be improved by deletion of any of the 15 items. Item-total correlation coefficients for all 15 items were be- tween 0.222 (item 12, "HF patients are discouraged from taking medications without food") and 0.514 (item 14, "HF patients had better take a high-salt diet").

\section{DisCUSSION}

Inadequate self-care behavior is one of the main causes of $\mathrm{HF}$ deterioration. Knowledge about HF is closely related to treatment adherence, and is key to the success of HF management intervention. ${ }^{10)}$ For accurate assessment of knowledge about HF, a valid and reliable instrument is needed. To the best of our knowledge, the instrument presented here is the first valid and reliable scale for assessment of HF knowledge in Japan. This instrument might be used not only to assess knowledge about their condition in patients with HF but also to gain insight into the effects of education and counseling on patients with $\mathrm{HF}$

In this study, items on the HF knowledge scale were based on information from the HF guidelines ${ }^{16-18)}$ and the results of previous studies, ${ }^{9,10,12,19-23)}$ and these were discussed in a focus group including a cardiologist and nurses with extensive HF knowledge. In the pilot study, patients reported no difficulty in answering or interpreting the questions. In addition, the time required to answer the questions was appropriate. From these results, we concluded that the content and face validity of the scale were adequately confirmed.

With regard to the construct validity of the HF knowledge scale, its one-dimensionality was comprehensively evaluated by the eigenvalue, scree plot, factor loading, and item contribution rate analyses. Two of the three items with low factor loadings were eliminated; item 12 regarding medication was retained considering the importance of assessing knowledge about medication. In previous studies, some patients exhibited 
Table III. Items Analysis for the HF Knowledge Scale $(n=187)$

\begin{tabular}{|c|c|c|c|}
\hline Item & & $\begin{array}{l}\text { Cronbach's } \alpha \text { if } \\
\text { item deleted }\end{array}$ & $\begin{array}{l}\text { Corrected } \\
\text { item-total } \\
\text { correlation }\end{array}$ \\
\hline 2 & Exchange of oxygen and carbon dioxide occurs in heart & 0.784 & 0.293 \\
\hline 3 & HF is a condition in which the heart is not able to pump blood through the body in sufficient amounts & 0.782 & 0.321 \\
\hline 4 & Difficulty in breathing and shortness of breath are symptoms of HF & 0.776 & 0.390 \\
\hline 5 & One of the symptoms when the lungs become congested with fluid is shortness of breath & 0.782 & 0.317 \\
\hline 6 & Some patients with severe HF become breathless when they lie flat and feel much better when they sit up & 0.773 & 0.422 \\
\hline 8 & Short-term weight gain is one of the signs of worsening HF & 0.773 & 0.423 \\
\hline 9 & Overwork and stress sometimes cause HF to get worse & 0.769 & 0.479 \\
\hline 10 & Sodium causes water retention & 0.772 & 0.437 \\
\hline 11 & Diuretics remove fluids from the body & 0.776 & 0.395 \\
\hline 12 & HF patients are discouraged from taking medications without food & 0.789 & 0.222 \\
\hline 13 & HF patients had better drink more water than healthy people & 0.771 & 0.450 \\
\hline 14 & HF patients had better take a high-salt diet & 0.766 & 0.514 \\
\hline 15 & Smoking is good for patients with HF because it promotes the circulation of blood & 0.768 & 0.488 \\
\hline 16 & HF patients should not perform exercise regardless of their severity of HF & 0.779 & 0.347 \\
\hline 17 & HF patients had better take a hot bath to promote blood circulation & 0.776 & 0.396 \\
\hline
\end{tabular}

HF indicates heart failure.

misunderstanding or knowledge deficiency on medication. ${ }^{11,22)}$ Such knowledge deficit is likely to lead to poor adherence to medication, which may cause deterioration of $\mathrm{HF}^{6,11,29)}$

$\mathrm{Ni}$, et al reported that greater knowledge was associated with receiving information or advice from health care providers. ${ }^{20)}$ Similarly, lower knowledge scores were observed for newly diagnosed patients, who had not yet received an educational intervention in the current study, which suggests that known-group validity was appropriate. Although we found a statistically significant difference in the knowledge scores between the two groups, the score of newly diagnosed patients was relatively high. This may reflect the fact that the questions are totally easy because we generated the need-to-know questions. Consequently, our knowledge scale may have an advantage for identifying patients who have poor HF knowledge. Instead, it would be difficult to rank patients who have a certain degree of knowledge. Therefore, careful attention should be paid to interpretation of the knowledge score.

As expected, the level of HF knowledge was positively correlated to self-care in the patients with HF included in this study. This finding agrees with previous findings, ${ }^{9-11,20)}$ and with Orem's theory, ${ }^{24)}$ which proposes that knowledge confers power, which in turn enables self-care. This similarity in results between this study and previous studies and with Orem's theory, suggests that the criterion validity of the scale was acceptable.

In terms of sensitivity, a significant difference was observed in scores before and after educational intervention in a subgroup of patients with HF. Thus, the new HF knowledge scale could be a useful tool to evaluate the effects of educational intervention in patients with HF. However, the sample utilized in this study was very small; thus, the difference between the clinical and statistical significance of this finding remains to be elucidated. Prospective studies are needed to determine a cutoff value of the new knowledge score in order to predict patient outcome.

Cronbach's alpha coefficient of the new HF knowledge scale was 0.79 . This value was almost satisfactory when judged against the recommended value of 0.70 or higher for internal consistency. ${ }^{30)}$ Item-total correlations of the scale ranged from 0.222 to 0.514 . Given that coefficients $>0.20$ are considered to be acceptable for item-total correlations, ${ }^{30)}$ the values observed here were acceptable.

Clinical and research implications: Poor self-care is well known to be a risk factor for adverse clinical outcomes in patients with $\mathrm{HF}^{4,7,11)}$ Knowledge is one of the important mediators of self-care practice. ${ }^{9,10,31)}$ Although this issue has been studied for decades, poor knowledge about their condition in patients with HF usually prevails; lack of regular assessment of patient knowledge contributes to this problem. The availability of the new HF knowledge scale developed here could benefit health care professionals in clinical practice, allowing them to assess deficiencies in HF knowledge and to identify areas in which patients need support. At the same time, patients who answer all the questions correctly may have a serious knowledge deficit on HF. We recommend use of the HF knowledge scale in combination with a good nursing assessment to assess the real knowledge deficit of HF patients. This new instrument may also allow evaluation of the effects of educational interventions on patient knowledge in future research.

The Dutch HF knowledge scale has been developed for research purposes and was widely utilized in previous studies; ${ }^{15)}$ meanwhile the scale presented here focuses more on HF management and self-care for use in clinical practice. In particular, the items on self-care were developed with reference to common misconceptions or concerns identified in patients with HF, as observed in other studies..$^{10,12,19-23)}$ For this reason, this scale may have greater power to assess patient knowledge more specifically.

Study limitations: Two limitations were observed in this study. First, the number of patients was relatively small, and enrollment was limited to patients with HF at two hospitals in Tokyo. Nonetheless, the study population was representative of Japanese patients with HF. In the registry study of Japanese HF patients, mean age was 67.8 years, males accounted for $67 \%$, and the ischemic etiology of the HF was in $25 \%$. ${ }^{2}$ Second, the test-retest reliability of the HF knowledge scale was not evaluated in this study, though the homogeneity of the scale was confirmed.

Conclusions: In this study, a HF knowledge scale was devel- 


\begin{tabular}{|c|c|c|c|c|}
\hline 1 & Exchange of oxygen and carbon dioxide occurs in heart & Yes & No & I don't know \\
\hline 2 & $\begin{array}{l}\text { HF is a condition in which the heart is not able to pump blood through } \\
\text { the body in sufficient amounts }\end{array}$ & Yes & No & I don't know \\
\hline 3 & Difficulty in breathing and shortness of breath are symptoms of $\mathrm{HF}$ & Yes & No & I don't know \\
\hline 4 & $\begin{array}{l}\text { One of the symptoms when the lungs become congested with fluid is } \\
\text { shortness of breath }\end{array}$ & Ye: & No & I don't know \\
\hline 5 & $\begin{array}{l}\text { Some patients with severe } \mathrm{HF} \text { become breathless when they lie flat and } \\
\text { feel much better when they sit up }\end{array}$ & Yes & No & I don't know \\
\hline 6 & Short-term weight gain is one of the signs of worsening $\mathrm{HF}$ & Yes & No & I don't know \\
\hline 7 & Overwork and stress sometimes cause HF to get worse & Yes & No & I don't know \\
\hline 8 & Sodium causes water retention & Yes & No & I don't know \\
\hline 9 & Diuretics remove fluids from the body & Yes & No & I don't know \\
\hline 10 & HF patients are discouraged from taking medications without food & Yes & No & I don't know \\
\hline 11 & HF patients had better drink more water than healthy people & Yes & No & I don't know \\
\hline 12 & HF patients had better take a high-salt diet & Yes & No & I don't know \\
\hline & $\begin{array}{l}\text { Smoking is good for patients with HF because it promotes the circulation } \\
\text { of blood }\end{array}$ & Yes & No & I don't know \\
\hline 14 & $\mathrm{HF}$ patients should not perform exercise regardless of their severity of $\mathrm{HF}$ & Yes & No & I don't know \\
\hline p & HF patients had better take a hot bath to promote blood circulation & Yes & No & I don't kno \\
\hline
\end{tabular}

Figure 2. The Japanese heart failure knowledge scale.

oped, and its psychometric properties were evaluated. The findings suggest that the new scale is useful for measurement of HF knowledge in patients with HF.

\section{ACKNOWLEDGMENTS}

We wish to express our sincere gratitude to Ms. Chikako Miura of the Sakakibara Heart Institute for helping with the recruitment of study participants and data collection.

\section{REFERENCES}

1. Okura Y, Ramadan MM, Ohno Y, et al. Impending epidemic: future projection of heart failure in Japan to the year 2055. Circ J 2008; 72: 489-91.

2. Shiba N, Watanabe J, Shinozaki T, et al. Analysis of chronic heart failure registry in the Tohoku district: third year follow-up. Circ J 2004; 68: 427-34.

3. Tsuchihashi-Makaya M, Hamaguchi S, Kinugawa S, et al. Characteristics and outcomes of hospitalized patients with heart failure and reduced vs preserved ejection fraction. Report from the Japanese Cardiac Registry of Heart Failure in Cardiology (JCARECARD). Circ J 2009; 73: 1893-900.

4. Annema C, Luttik ML, Jaarsma T. Reasons for readmission in heart failure: Perspectives of patients, caregivers, cardiologists, and heart failure nurses. Heart Lung 2009; 38: 427-34.

5. Kollipara UK, Jaffer O, Amin A, et al. Relation of lack of knowledge about dietary sodium to hospital readmission in patients with heart failure. Am J Cardiol 2008; 102: 1212-5.

6. Fitzgerald AA, Powers JD, Ho PM, et al. Impact of medication nonadherence on hospitalizations and mortality in heart failure. J Card Fail 2011; 17: 664-9.

7. van der Wal MH, van Veldhuisen DJ, Veeger NJ, Rutten FH, Jaarsma T. Compliance with non-pharmacological recommendations and outcome in heart failure patients. Eur Heart J 2010; 31: 1486-93.

8. McAlister FA, Stewart S, Ferrua S, McMurray JJ. Multidisciplinary strategies for the management of heart failure patients at high risk for admission: a systematic review of randomized trials. J Am Coll Cardiol 2004; 44: 810-9. (Review)

9. Artinian NT, Magnan M, Sloan M, Lange MP. Self-care behaviors among patients with heart failure. Heart Lung 2002; 31: 161-72.

10. van der Wal MH, Jaarsma T, Moser DK, Veeger NJ, van Gilst WH, van Veldhuisen DJ. Compliance in heart failure patients: the importance of knowledge and beliefs. Eur Heart J 2006; 27: 43440.
11. Riegel B, Moser DK, Anker SD, et al. State of the science: promoting self-care in persons with heart failure: a scientific statement from the American Heart Association. Circulation 2009; 120: 1141-63. (Review)

12. van der Wal MH, Jaarsma T, Moser DK, van Gilst WH, van Veldhuisen DJ. Qualitative examination of compliance in heart failure patients in The Netherlands. Heart Lung 2010; 39: 121-30.

13. Jackson JL, Emery CF. Illness knowledge moderates the influence of coping style on quality of life among women with congestive heart failure. Heart Lung 2011; 40: 122-9.

14. Reilly CM, Higgins M, Smith A, et al. Development, psychometric testing, and revision of the Atlanta Heart Failure Knowledge Test. J Cardiovasc Nurs 2009; 24: 500-9.

15. van der Wal MH, Jaarsma T, Moser DK, van Veldhuisen DJ. Development and testing of the Dutch Heart Failure Knowledge Scale. Eur J Cardiovasc Nurs 2005; 4: 273-7.

16. Hunt SA, Abraham WT, Chin MH, et al. 2009 focused update incorporated into the ACC/AHA 2005 Guidelines for the Diagnosis and Management of Heart Failure in Adults: a report of the American College of Cardiology Foundation/American Heart Association Task Force on Practice Guidelines: developed in collaboration with the International Society for Heart and Lung Transplantation. Circulation 2009; 119: e391-479. (Review)

17. McMurray JJ, Adamopoulos S, Anker SD, et al. ESC Guidelines for the diagnosis and treatment of acute and chronic heart failure 2012: The Task Force for the Diagnosis and Treatment of Acute and Chronic Heart Failure 2012 of the European Society of Cardiology. Developed in collaboration with the Heart Failure Association (HFA) of the ESC. Eur Heart J 2012; 33: 1787-847.

18. The Japanese Circulation Society. Guidelines for treatment of chronic heart failure (JCS 2010). Available at: http://www.j-circ. or.jp/guideline/pdf/JCS2010_matsuzaki_h.pdf. Accessed November 12, 2012.

19. Riegel B, Carlson B. Facilitators and barriers to heart failure selfcare. Patient Educ Couns 2002; 46: 287-95.

20. Ni H, Nauman D, Burgess D, Wise K, Crispell K, Hershberger RE. Factors influencing knowledge of and adherence to self-care among patients with heart failure. Arch Intern Med 1999; 159: 1613-9.

21. van der Wal MH, Jaarsma T, van Veldhuisen DJ. Non-compliance in patients with heart failure; how can we manage it? Eur J Heart Fail 2005; 7: 5-17. (Review)

22. Kato N, Kinugawa K, Sano M, et al. Development of self-care educational material for patients with heart failure in Japan: a pilot study. Nurs Health Sci 2012; 14: 156-64.

23. Carlson B, Riegel B, Moser DK. Self-care abilities of patients with heart failure. Heart Lung 2001; 30: 351-9.

24. Orem DE. Nursing concepts of practice. 5 th ed. St. Louis: Mosby; 1995.

25. Kato N, Ito N, Kinugawa K, Kazuma K. Validity and reliability of the Japanese version of the European Heart Failure Self-Care Behavior Scale. Eur J Cardiovasc Nurs 2008; 7: 284-9.

26. Jaarsma T, Strömberg A, Mårtensson J, Dracup K. Development and testing of the European Heart Failure Self-Care Behaviour Scale. Eur J Heart Fail 2003; 5: 363-70.

27. Matsuo S, Imai E, Horio M, et al. Revised equations for estimated GFR from serum creatinine in Japan. Am J Kidney Dis 2009; 53: 982-92.

28. Moser DK, Doering LV, Chung ML. Vulnerabilities of patients recovering from an exacerbation of chronic heart failure. Am Heart J 2005; 150: 984.

29. Wu JR, Moser DK, Chung ML, Lennie TA. Objectively measured, but not self-reported, medication adherence independently predicts event-free survival in patients with heart failure. J Card Fail 2008; 14: 203-10.

30. David LS, Geoffrey RN. Health Measurement Scales: a practical guide to their development and use. 3rd ed. New York: Oxford University Press; 2003.

31. Jaarsma T. Inter-professional team approach to patients with heart failure. Heart 2005; 91: 832-8. (Review) 